\title{
Regression activity that is naturally present in vitreous becomes ineffective as patients develop proliferative diabetic retinopathy
}

\author{
J. Aranda • R. Motiejunaite • P. Silva • L. P. Aiello • \\ A. Kazlauskas
}

Received: 12 November 2012 / Accepted: 12 February 2013 / Published online: 19 March 2013

(C) Springer-Verlag Berlin Heidelberg 2013

\begin{abstract}
Aims/hypothesis The realisation that targeting agents in the vitreous is an effective approach to treating patients with diabetic retinopathy (DR) has increased awareness that changes in the composition/bioactivity of the vitreous is a contributor to the pathogenesis of DR. The overall goal of this study was to test the hypothesis that the vitreous has regression activity, and that lysophosphatidic acid (LPA) contributes to such activity. LPA is a bioactive phospholipid present in many biological fluids, and has been recently appreciated for its ability to promote regression of blood vessels. Methods Vitreous-mediated regression was monitored on tubes organised from primary retinal endothelial cells or neovessels that sprouted from retinal explants. LPA was quantified radioenzymatically.

Results Bovine and human vitreous promoted regression of retinal explant vessels and of tubes organised from primary retinal endothelial cells. LPA was a substantial component of this regression activity. Comparing the regression activities of vitreous from patients with different stages of DR revealed that, as patients developed proliferative diabetic
\end{abstract}

Electronic supplementary material The online version of this article (doi:10.1007/s00125-013-2884-2) contains peer-reviewed but unedited supplementary material, which is available to authorised users.

J. Aranda $\cdot$ R. Motiejunaite $\cdot$ A. Kazlauskas $(\bowtie)$

Schepens Eye Research Institute, Massachusetts Eye and Ear

Infirmary, 20 Staniford Street,

Boston, MA 02114, USA

e-mail: Andrius_kazlauskas@meei.harvard.edu

R. Motiejunaite

Department of Biochemistry and Biophysics, Vilnius University,

Vilnius, Lithuania

P. Silva • L. P. Aiello

Joslin Diabetes Center, Boston, MA, USA retinopathy (PDR), vitreous lost its ability to promote regression, even though the amount of LPA did not change. The underlying mechanism was a PDR-vitreousmediated insensitivity to LPA, which could be overcome pharmacologically.

Conclusions/interpretation Our findings suggest that a decline in the responsiveness to regression factors such as LPA, which are naturally present in the vitreous, contributes to the pathogenesis of PDR.

Keywords Basic science $\cdot$ Endothelium $\cdot$ Microvascular disease $\cdot$ Retinopathy

$\begin{array}{ll}\text { Abbreviations } \\ \text { ATX } & \text { Autotaxin } \\ \text { BREC } & \text { Bovine retinal endothelial cell } \\ \text { BrP-LPA } & \begin{array}{l}\text { 1-Bromo-3(S)-hydroxy-4-[(palmitoyloxy)butyl] } \\ \text { phosphonate }\end{array} \\ \text { BVi } & \text { Bovine vitreous } \\ \text { DR } & \text { Diabetic retinopathy } \\ \text { EBM } & \text { Endothelial cell basal medium } \\ \text { ERK } & \text { Extracellular signal-regulated kinase } \\ \text { HVi } & \text { Human vitreous } \\ \text { LPA } & \text { Lysophosphatidic acid } \\ \text { LPAAT } & \text { Lysophosphatidic acid acyl-transferase } \\ \text { LPP1 } & \text { Lipid phosphate phosphatase-1 } \\ \text { NAC } & \text { N-Acetyl cysteine } \\ \text { NPDR } & \text { Non-proliferative diabetic retinopathy } \\ \text { PA } & \text { Phosphatidic acid } \\ \text { PDR } & \text { Proliferative diabetic retinopathy } \\ \text { QPDR } & \text { Quiescent proliferative diabetic retinopathy } \\ \text { ROCK } & \text { Rho-associated protein kinase } \\ \text { ROS } & \text { Reactive oxygen species } \\ \text { SFK } & \text { Src family kinase } \\ \text { VEGF-A } & \text { Vascular endothelial growth factor A }\end{array}$




\section{Introduction}

Diabetes mellitus currently afflicts 366 million people worldwide, and its prevalence is sharply increasing. It is predicted that 552 million people will be suffering from diabetes in 2030 [1]. Unfortunately, most people (80\%) with diabetes live in low- and middle-income countries that are poorly prepared to manage the costs brought by diabetes and its complications. There is no cure for diabetes and available treatments have only limited success in mitigating the resulting complications. Even in industrialised countries such as the USA, diabetes is the leading cause of new cases of blindness among adults [2].

Diabetic retinopathy (DR) is one of the microvascular complications of diabetes mellitus. The majority of patients with diabetes mellitus eventually develop some degree of DR [3]. The first of the two stages of DR is non-proliferative diabetic retinopathy (NPDR), associated with the death of pericytes that reside within retinal capillaries [2, 4-6]. Pericyte dropout compromises the functionality of retinal microvessels and thereby creates hypoxia, which stimulates production of pro-angiogenic agents such as vascular endothelial growth factor A (VEGF-A) and erythropoietin [7, 8]. These agents drive angiogenesis, which is a quintessential feature of the second stage of DR, called proliferative diabetic retinopathy (PDR) $[6,9]$.

As our appreciation of PDR pathogenesis has improved, new therapies have emerged (e.g. anti-VEGF-A therapy) $[3,10]$. While these alternatives are effective in some patients, the fact that not all respond underscores our incomplete understanding of PDR pathogenesis [3, 10-14].

Reports that appeared in the late 1970s and early 1980s documented that the vitreous from humans and experimental animals suppressed the angiogenic process in a variety of models [15-17]. These observations suggested that vitreous contained anti-angiogenic agents that contributed to angiogenic homeostasis of the retinal vasculature. The identity of these agents, and their mechanism of action (prevention of the growth of new vessels vs induction of regression) have remained unanswered.

Lysophosphatidic acid (LPA) is a bioactive phospholipid present in many biological fluids [18-20]. It is produced by the action of autotaxin (ATX) on its substrate lysophosphatidylcholine (LPC) [18-20]. ATX and LPA have contrasting effects on angiogenesis. On one hand, ATX and LPA are key players in early vascular development [21], and overproduction of ATX or LPA receptors facilitates tumour formation and angiogenesis [22-24]. On the other, ATX and LPA promote regression. ATX overproduction accelerates regression of unstable vascular beds such as hyaloid vessels [25, 26]. Furthermore, LPA promotes migration of endothelial cells and can induce disorganisation and regression of tubes organised from primary retinal endothelial cells
$[25,26]$. Taken together, these findings indicate that LPA is an angiomodulator that can promote either the formation or the regression of blood vessels, depending on the context.

We recently reported that diabetes influences the responsiveness of endothelial cells to LPA. While retinal neovessels readily regressed when exposed to LPA, retinal neovessels from diabetic mice were resistant to LPA-mediated regression $[25,26]$. The underlying mechanism appeared to involve high-glucose-induced elevation of reactive oxygen species (ROS) that engaged a signalling pathway that resulted in unresponsiveness to LPA $[25,26]$. These findings indicated that diabetes mellitus disrupted the responsiveness of retinal endothelial cells to LPA.

In light of the results discussed above, the goals of the present study were: (1) to test the hypothesis that LPA contributes to the regression activity of the vitreous; and (2) to investigate whether progression to PDR influences the regression activity of vitreous.

\section{Methods}

Antibodies and reagents Anti-mouse and anti-rabbit horseradish-peroxidase-conjugated antibodies were from Santa Cruz Biotechnology (Santa Cruz, CA, USA). The (extracellular signal-regulated kinase (ERK) kinase (MEK)) inhibitor (U0126) was obtained from Cell Signaling Technology (Beverly, MA, USA). The pan-LPA receptor antagonist 1-bromo-3(S)-hydroxy-4-[(palmitoyloxy)butyl] phosphonate (BrP-LPA) and Ki16425 were from Echelon Biosciences (Salt Lake City, UT, USA) and Cayman Chemical (Ann Arbor, MI, USA), respectively. LPA (oleoyl-L- $\alpha$-lysophosphatidic acid sodium salt, L7260), the antibodies directed against the FLAG epitope tag (F1804) and lipid phosphate phosphatase-1 (LPP1; AV42146), and the antioxidants $N$-acetylcysteine (NAC) and Tiron (A7250 and D7389, respectively) were from Sigma (St Louis, MO, USA). Catalase (1872) was from Worthington Biochemical (Lakewood, NJ, USA). The Src inhibitor (Su6656) was purchased from Calbiochem (Gibbstown, NJ, USA).

Retinal explants Explants were isolated and cultured as described [26, 27]. Briefly, wild-type C57BL6 mice were killed, and their retinas were isolated and cut into $1-2 \mathrm{~mm}$ pieces. The tissue was placed in a collagen sandwich (80\% (wt./vol.) collagen [Advanced Biomatrix, San Diego, CA, USA], $20 \mathrm{mmol} / 1 \mathrm{HEPES}$ [Lonza, Hopkinton, MA, USA], $0.5 \mu \mathrm{g} / \mathrm{ml}$ fibronectin and laminin [both from Sigma], $2 \mathrm{mg} / \mathrm{ml}$ $\mathrm{NaHCO}_{3}$ and $0.02 \mathrm{mmol} / \mathrm{l} \mathrm{NaOH}$ ) that was covered with endothelial cell basal medium (EBM; Lonza). Medium was supplemented with 10\% (vol./vol.) horse serum (Lonza), $12 \mu \mathrm{g} / \mathrm{ml}$ bovine brain extract (Hammond Cell Tech, Windsor, CA, USA) and $80 \mathrm{U} / \mathrm{ml}$ penicillin-streptomycin, 
plus $25 \mathrm{ng} / \mathrm{ml}$ VEGF-A. Explants were maintained at $37^{\circ} \mathrm{C}$ with $5 \%$ (vol./vol.) $\mathrm{CO}_{2}$. The Institutional Animal Care and Use Committee of Schepens Eye Research Institute approved all procedures. The mice were maintained following the Principles of Laboratory Animal Care from the National Institutes of Health.

Retinal explant vessel regression Vessel formation was typically observed 2 weeks after placing retinal explants in culture as described above. This time point was designated time 0 , and vessels were photographed at this time point with a Nikon (Melville, NY, USA) TE-2000S inverted microscope. Subsequently, treatment was added and replenished twice a day [25]. Two days after the initiation of treatment, the explants were photographed again. All photographs were exported as tagged image files (TIFFs) into NIH Image $\mathrm{J}$ (http://rsbweb.nih.gov/ij/). The total vessel length was measured before and after treatment. A decrease in vessel length indicated regression. Normalised tube length was the ratio of tube lengths before and after treatment $[26,27]$. The data presented are the mean of triplicates from at least three independent experiments.

Cell culture Primary bovine retinal endothelial cells (BRECs) were isolated as described [27]. The cells were cultured in EBM (Lonza) supplemented as described for the retinal explants. Primary retinal endothelial cells were used between passages 10 and 13 . All cells were maintained at $37^{\circ} \mathrm{C}$ with $5 \%$ (vol./vol.) $\mathrm{CO}_{2}$.

Vitreous Bovine eyes were obtained from the local slaughterhouse. The eyes were rinsed for $1 \mathrm{~h}$ in a 1:5 dilution of Povidone-iodine (CVS Pharmacy, Boston, MA, USA). Next, the eyes were rinsed four times in sterile PBS and dried on paper towels previously rinsed with $70 \%$ (vol./vol.) ethanol. The tissue surrounding the eyeball was carefully removed. The cornea was excised along the limbus, and the lens was carefully removed with forceps. The vitreous was isolated and centrifuged for $10 \mathrm{~min}$ at $1,500 \mathrm{~g}$. The supernatant fraction was aliquoted and stored at $-80^{\circ} \mathrm{C}$.

The Internal Review Board of the Joslin Diabetes Institute approved the collection of human vitreous used in this study (Protocol 2002-P-0002237/7). Patients underwent pars plana vitrectomy. The samples of ocular fluid were collected in sterile tubes, placed immediately on ice, clarified by centrifugation at $16,000 \mathrm{~g}$ for $5 \mathrm{~min}$ at $4^{\circ} \mathrm{C}$, and rapidly frozen at $-80^{\circ}$ C. Patient information was collected and recorded in a securepassword-protected database. All samples were stored at Joslin Diabetes Center, where they were catalogued and labelled in an anonymous fashion. In all cases, written informed consent was obtained from donors. The human samples used in this study were from: 13 patients without diabetes (No DM; patients had been admitted for non-angiogenic eye disease such as rhegmatogenous retinal detachment, macular hole or subluxed intraocular lens); six patients with diabetes but no visible signs of DR (DM no DR); seven patients at the NPDR stage; 19 patients with PDR; and seven patients with quiescent PDR (QPDR). This last group were PDR patients who had been treated (typically with laser) and the pathological retinal neovessels had regressed. The sample size was 80-110 $\mu \mathrm{l}$.

Collagen sandwich tube formation assay The tube assay was performed as described $[26,28]$. BRECs were placed in a collagen gel sandwich and treated overnight with EBM supplemented with VEGF-A $(2.5 \mathrm{ng} / \mathrm{ml})$. Tube formation was observed $12 \mathrm{~h}$ later using a Nikon TE-2000S microscope. All treatments were performed in fresh media supplemented with VEGF-A. To observe the effect of endogenous LPA, the assay was performed in EBM containing 10\% (vol./vol.) horse serum (Lonza). Low serum concentration diminishes the ability of BRECs to regress as they do in the presence of $10 \%$ horse serum $[25,26]$. Thus, the effect of exogenous LPA $(1-10 \mu \mathrm{mol} / \mathrm{l})$ was studied in EBM with $0.5 \%$ (vol./vol.) horse serum.

Tube regression evaluation Regression was evaluated as previously described $[25,27]$. Tubes were photographed $12 \mathrm{~h}$ after the initial addition of VEGF-A. At this time tube formation is maximal $[26,28,29]$, and it was designated as time 0 . Next, the medium was removed and treatments were added in the presence of VEGF-A. After $4 \mathrm{~h}$, the fields that were initially documented were re-photographed. The photograph files were exported to NIH Image J for analysis. A decrease in tube length was scored as regression; normalised tube length was calculated as the ratio of final and initial tube lengths [25]. Each experimental condition was evaluated in triplicate, and all experiments were independently repeated at least three times.

Immunopurification of lipid phosphate phosphatase The cDNA coding for the FLAG-tagged LPP1 protein (also known as PPAP2A) cDNA [25] was subcloned into the retroviral pLXSN vector, and transfected into $293 \mathrm{GPG}$ cells. Virus-containing media was collected for 5 days and concentrated at $4^{\circ} \mathrm{C}(25,000 \mathrm{~g}$ for $90 \mathrm{~min})$ and used as previously described $[26,28]$ to infect NIH $3 \mathrm{~T} 3$ cells that were subsequently selected based on G418 $(1 \mathrm{mg} / \mathrm{ml})$ resistance. Control cells were infected with an empty pLXSN vector. The cells were allowed to proliferate to $80-90 \%$ confluence in a $15 \mathrm{~cm}$ tissue culture dish. Cell lysates were prepared by the addition of lysis buffer $(20 \mathrm{mmol} / 1$ Tris, $\mathrm{pH} 8$, $150 \mathrm{mmol} / 1 \mathrm{NaCl}, 1 \mathrm{mmol} / \mathrm{l}$ dithiothreitol, $1 \%$ (wt./vol.) deoxycholic acid, $0.5 \%$ (wt./vol.) SDS, 1\% (vol./vol.) Nonidet P-40, and protease inhibitors $[2 \mu \mathrm{g} / \mathrm{ml}$ aprotinin and $10 \mu \mathrm{g} / \mathrm{ml}$ phenylmethylsulfonyl fluoride]) and incubated for $1 \mathrm{~h}$ at $4^{\circ} \mathrm{C}$ with agitation. The samples were centrifuged, and the supernatant fraction was incubated 
a

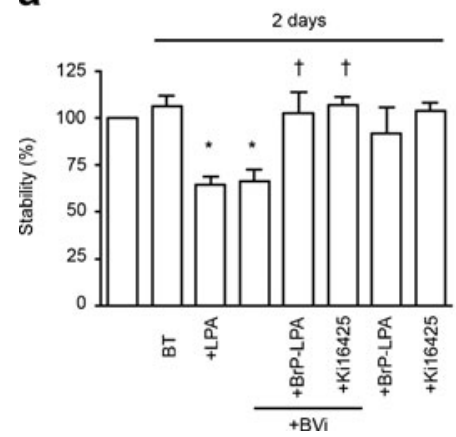

b

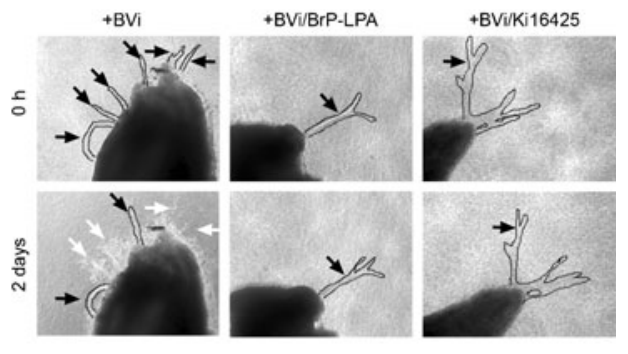

Fig. 1 Regression of retinal explant vessels was induced by BVi in an LPA-receptor-dependent manner. Retinas were subjected to a retinal explant assay as described in the Methods section. Lumenised vessels composed of endothelial cells developed in 2 weeks [26]. LPA $(1 \mu \mathrm{mol} / \mathrm{l})$ or BVi was added for 2 days; the treatments were renewed every $12 \mathrm{~h}$. The vessel length was measured before and after the indicated treatments. Data shown are the means \pm SEM of four independent experiments. (a) Treatment with $\mathrm{BVi}$ induced regression comparable with that mediated by LPA. Addition of the LPA receptor inhibitors BrP-LPA $(10 \mu \mathrm{mol} / \mathrm{l})$ or
Ki16425 (5 $\mu \mathrm{mol} / \mathrm{l})$ in combination with BVi inhibited regression. Addition of the inhibitors alone had no effect. The percentage stability is the ratio of vessel lengths before and after treatment. ${ }^{*} p<0.05$ vs explants treated with buffer; ${ }^{\dagger} p<0.05$ vs explants treated with $\mathrm{BVi}$; BT, buffertreated. (b) Representative photographs of vessels treated with BVi alone or BVi treatment in the presence of BrP-LPA or Ki16425. Regressing vessels are indicated with white arrows. Stable vessels are indicated with black arrows. All vessels were digitally outlined to facilitate their visualisation (solid lines). Magnification is $\times 100$ for all images a

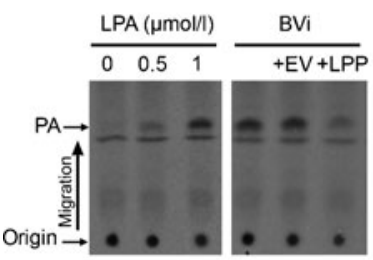

b

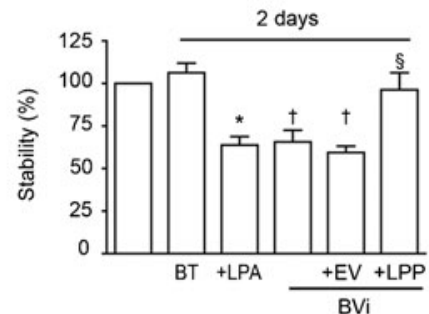

c
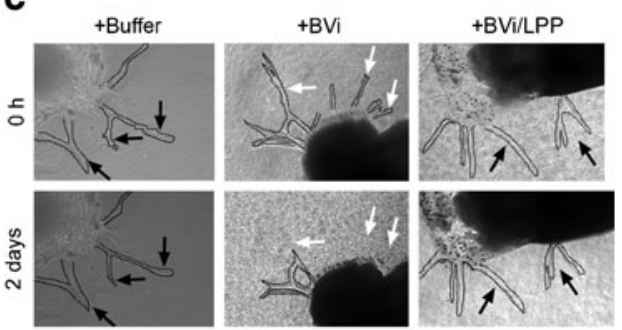

d

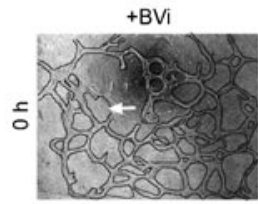

$+\mathrm{BVi} / \mathrm{mock}$
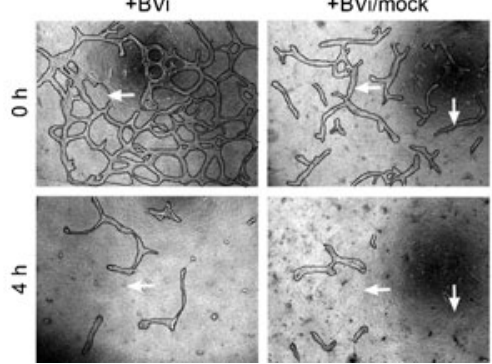

$+B V i / L P P$
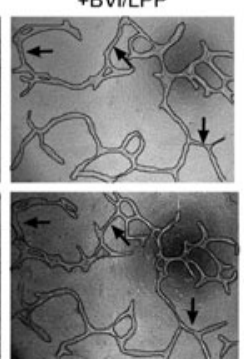

e

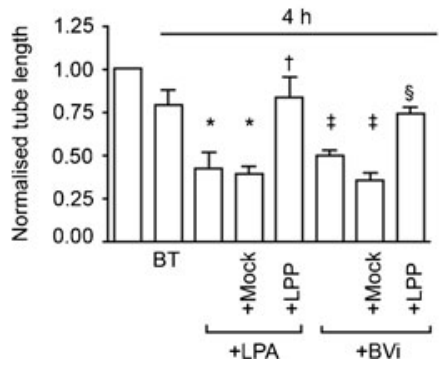

Fig. 2 LPA was present in BVi and accounted for a major portion of the regression activity. (a) The amount of LPA in BVi was determined using a radioenzymatic LPA assay, which is described in the Methods. In this assay LPA is converted into radioactively labelled PA [30]. The reaction products were resolved by thin-layer chromatography. Compared with LPA standards BVi contained $0.8 \pm 0.13 \mu \mathrm{mol} / 1 \mathrm{LPA}$. LPA present in BVi was cleared by treatment with isolated LPP. Mock treatment (EV; an immunoprecipitate prepared from cells that did not overproduce LPP1) had no effect on the concentration of vitreal LPA. (b,c) Retinal explants were isolated and cultured as described in Fig. 1. LPA $(1 \mu \mathrm{mol} / \mathrm{l})$ and BVi induced comparable regression. Clearing LPA from vitreous eliminated regression activity. Black and white arrows indicate stable and regressing vessels, respectively. Four independent experiments showed similar results. Data shown are means $\pm \operatorname{SEM}(n=4){ }^{*} p<0.05$ compared with buffer-treated (BT) explants; ${ }^{\dagger} p<0.05$ compared with buffer-treated explants, no significant difference compared with LPA treatment; ${ }^{{ }} p<0.05$ compared with
LPA, BVi and empty-vector (EV) treated BVi. (d,e) Primary BRECs were subjected to a collagen sandwich tube formation assay in low serum $(0.5 \%$ [vol./vol.]); this assay is described in the Methods. Tube formation was photographed $12 \mathrm{~h}$ after the addition of VEGF-A $(2.5 \mathrm{ng} / \mathrm{ml})$, the indicated treatments were added and tubes were re-photographed after $4 \mathrm{~h}$. Tubes regressed in response to either $\mathrm{BVi}$ or mock-treated $\mathrm{BVi}$ (white arrows in images), whereas they remained stable with LPP-treated BVi (black arrows). Magnification is $\times 100$. Tubes were digitally outlined to facilitate their visualisation. The results when LPA $(1 \mu \mathrm{mol} / \mathrm{l})$ was added instead of $\mathrm{BVi}$ are also shown in (e). Data shown are means $\pm \operatorname{SEM}(n=4)$. Normalised tube length is the ratio of final and initial tube lengths. ${ }^{*} p<0.05$ compared with buffer-treated tubes; ${ }^{\dagger} p<0.05$ compared with tubes treated with LPA or mock treated. ${ }^{\star} p<0.05$ compared with buffer-treated (BT) tubes; ${ }^{\S} p<0.05$ compared with BVi- and mock-treated tubes. Normalised tube length is the ratio of final and initial tube lengths 
overnight with the anti-FLAG antibody $(1 \mu \mathrm{g})$ at $4^{\circ} \mathrm{C}$. The next morning $100 \mu \mathrm{l}$ of protein A-agarose beads (sc-2001, Santa Cruz, CA, USA) were added to samples for $3 \mathrm{~h}$ at $4^{\circ} \mathrm{C}$ with agitation. After centrifugation, the beads were washed three times with PBS and stored in small aliquots at $-80^{\circ} \mathrm{C}$. The procedure for the mock immunoprecipitation was the same, but performed with lysates from 3T3 cells infected with empty expression vector.

LPA assay The concentration of LPA in vitreous samples was measured using a previously reported radioenzymatic method [30-32]. Briefly, lipids were extracted with butanol and incubated in a reaction mixture containing recombinant LPA acyl-transferase (LPAAT) and $\left[{ }^{14} \mathrm{C}\right]$ oleoyl-CoA (Perkin-Elmer, Waltham, MA, USA). Subsequently, the reaction products were resolved by thin-layer chromatography. Conversion of LPA into radioactively labelled phosphatidic acid (PA) by LPAAT was identified by autoradiography and densitometrically quantified. The correct position of LPA and PA on the thin-layer plate was determined by running commercially obtained LPA and PA standards in parallel [26, 33].

Image post-processing As in previous studies [26], we enhanced the visibility of the tubes and vessels as follows. Representative pictures were imported to Adobe Photoshop CS3, and the perimeter of the vascular structures was delineated using the pencil function. The corresponding figures without the enhancement (outlining) are presented in the electronic supplementary material (ESM) Fig. 1.

Statistics Data were analysed using GraphPad Prism (www.graphpad.com/) version 4 software. Experiments were analysed using ANOVA. Differences were considered statistically significant if $p<0.05$.

\section{Results}

Bovine vitreous promoted regression in an LPA-dependent manner Reports that appeared in the late 1970s or early 1980s documented that vitreous from humans or experimental animals suppressed neovascularisation in a variety of models [15-17]. As suppression of neovascularisation may result from preventing angiogenesis and/or inducing vessel regression, we sought to extend these studies by testing the hypothesis that vitreous promoted regression of retinal neovessels. To this end, we added bovine vitreous (BVi) to neovessels that sprouted from mouse retinal explants. These vessels are lumenised and composed of endothelial cells [26]. As shown in Fig. 1, vitreous induced regression of the majority of vessels (white arrows). Similarly, BVi

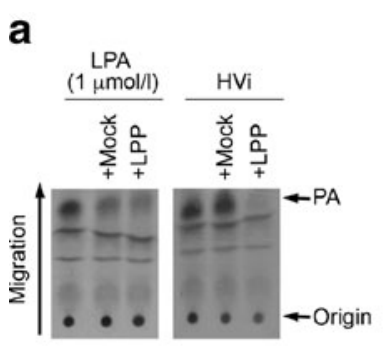

b
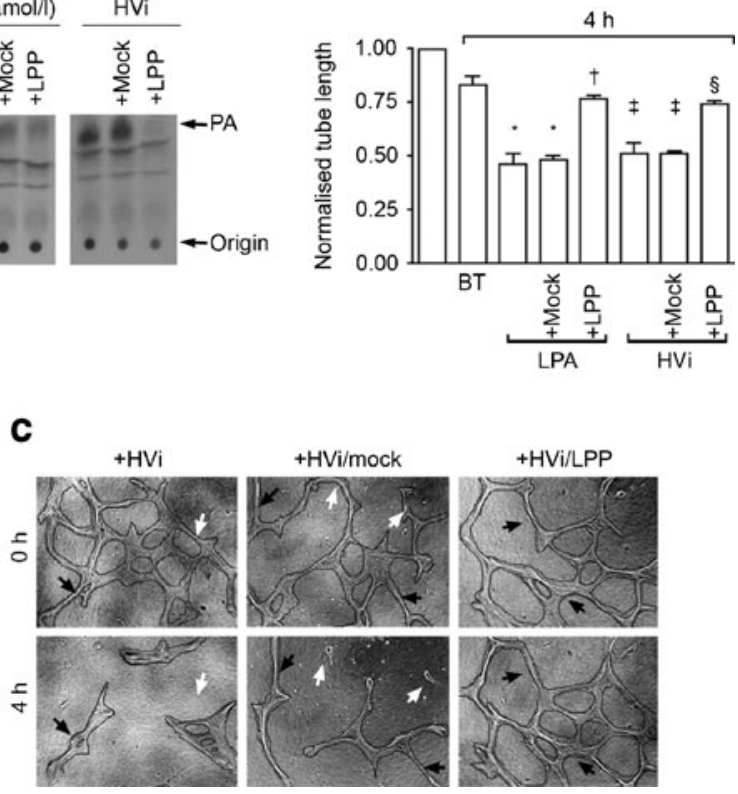

Fig. 3 LPA was present in HVi and was critical for its regression activity. (a) LPA was detected in HVi from patients with non-angiogenic retinal disease (as described in Table 1). (b,c) Control or HVi that was cleared of LPA was tested for its ability to induce regression of tubes as described in the legend to Fig. 2. Similarly to BVi, HVi contained LPA, which was an essential component of vitreous regression activity. Normalised tube length is the ratio of final and initial tube lengths. ${ }^{*} p<0.05$ compared with buffer-treated (BT) tubes; ${ }^{\dagger} p<0.05$ compared with tubes treated with LPA or mock treatment; ${ }^{\ddagger} p<0.05$ compared with buffer-treated tubes; ${ }^{\S} p<0.05$ compared with HVi- and mock-treated tubes

induced regression of tubes organised from primary retinal endothelial cells (Fig. 2d). We conclude that one of the ways in which vitreous is anti-angiogenic is by promoting regression and that it acts at the level of endothelial cells in the absence of all other retinal cell types.

Table 1 Characteristics of vitreous that was analysed

\begin{tabular}{llll}
\hline $\begin{array}{l}\text { Vitreous } \\
\text { specimen }\end{array}$ & $\begin{array}{l}\text { Number of } \\
\text { samples }\end{array}$ & $\begin{array}{l}\text { Patient age } \\
\text { (years) }\end{array}$ & $\begin{array}{l}\text { Duration of diabetes } \\
\text { (years) }\end{array}$ \\
\hline No DM & 12 & $65-75$ & $\begin{array}{c}\text { NA, admitted for non- } \\
\text { angiogenic eye disease }\end{array}$ \\
DM no DR & 4 & $65-74$ & $17-61$ \\
NPDR & 9 & $72-84$ & $10-76$ \\
PDR & 19 & $29-83$ & $13-46$ \\
QPDR & 9 & $47-67$ & $\begin{array}{c}9-16 \text { (admitted for non- } \\
\text { angiogenic eye disease) }\end{array}$
\end{tabular}

As the amount of vitreous obtained from patients varied and was typically insufficient to perform all analyses, vitreous from patients with the same stage of DR was often (but not always, and in such cases noted) pooled prior to analysis

NA, not applicable 
What is in vitreous that is responsible for regression activity? We previously reported that LPA promotes regression of tubes organised from primary retinal endothelial cells [25], of neovessels that sprout from retinal explants (as in Fig. 1) [26] and of blood vessels in vivo [25]. Consequently, we considered the contribution of LPA to the regression activity in vitreous. Figure 2 shows that vitreous contained LPA $(0.81 \pm 0.13 \mu \mathrm{mol} / \mathrm{l})$; this amount is in the range that suffices to induce regression [26]. Furthermore, two chemically distinct antagonists of LPA receptors prevented vitreous-induced regression (Fig. 1). Finally, vitreous from which LPA was enzymatically cleared lost its ability to promote regression of neovessels and tubes (Fig. 2). These data indicate that LPA accounts for a substantial portion of regression activity in vitreous.

Human vitreous contained LPA, which contributed to regression activity We also tested if human vitreous (HVi) from donors had regression activity, and whether the activity depended on LPA. The data shown in Fig. 3 reveal a very similar picture to $\mathrm{BVi}$ : HVi promoted regression and it contained LPA $(0.79 \pm 0.15 \mu \mathrm{mol} / \mathrm{l})$ at a level sufficient for regression
[26]. Enzymatically removing the LPA diminished the regression activity of HVi. Because of the limited availability of $\mathrm{HVi}$, we did not test its ability to promote regression of retinal neovessels; regression of neovessels took longer ( 2 days vs $4 \mathrm{~h}$ ) and hence required more vitreous than regression of tubes. We conclude that HVi also contains LPA that is required for its intrinsic regression activity.

Vitreous from patients with PDR failed to promote regression, even though it contained LPA The presence of regression activity in vitreous suggested that accumulation of neovessels in vitreous of patients with PDR might result not only from the well-known rise in pro-angiogenic agents $[7,8]$, but also because of a decline in regression activity. This idea predicts that vitreous from patients with PDR would have lower regression activity than patients with non-angiogenic stages of DR. To test this idea we compared the bioactivity of vitreous from patients with different stages of DR (Table 1). Vitreous from No DM, DM no DR, NPDR and even QPDR patients promoted regression, whereas PDR vitreous did not (Fig. 4a-c). While these bioactivity results predicted that PDR vitreous would have little LPA, this was not the case;
Fig. 4 PDR vitreous contained LPA, but failed to promote regression. (a) Tubes were formed as described in the legend to Fig. 2. Vitreous from patients with the indicated stage of DR was added after tubes had formed and regression was assessed $4 \mathrm{~h}$ later.

Representative photos are shown in (c). Three specimens of each type were analysed. Data shown are means \pm SEM. ${ }^{*} p<0.05$ vs buffer-treated (BT) control. (b) Regression activity for non-angiogenic vitreous specimens. Samples from no DM and DM no DR patients induced significant regression $\left({ }^{*} p<0.05\right.$ vs control) of the tubes; $n=3$ and $n=4$, respectively. Normalised tube length is the ratio of final and initial tube lengths. (d) LPA was quantified in vitreous from patients with different stages of DR ( $n=3$ for NPDR and QPDR, $n=4$ for PDR) or no DM $(n=3)$. (e) Quantification of vitreous LPA. Data shown are means \pm SEM. There were no differences in the level of LPA between the groups
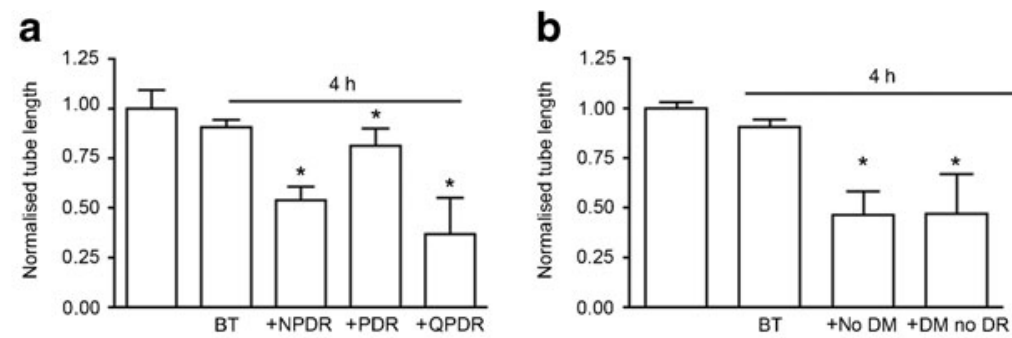

C
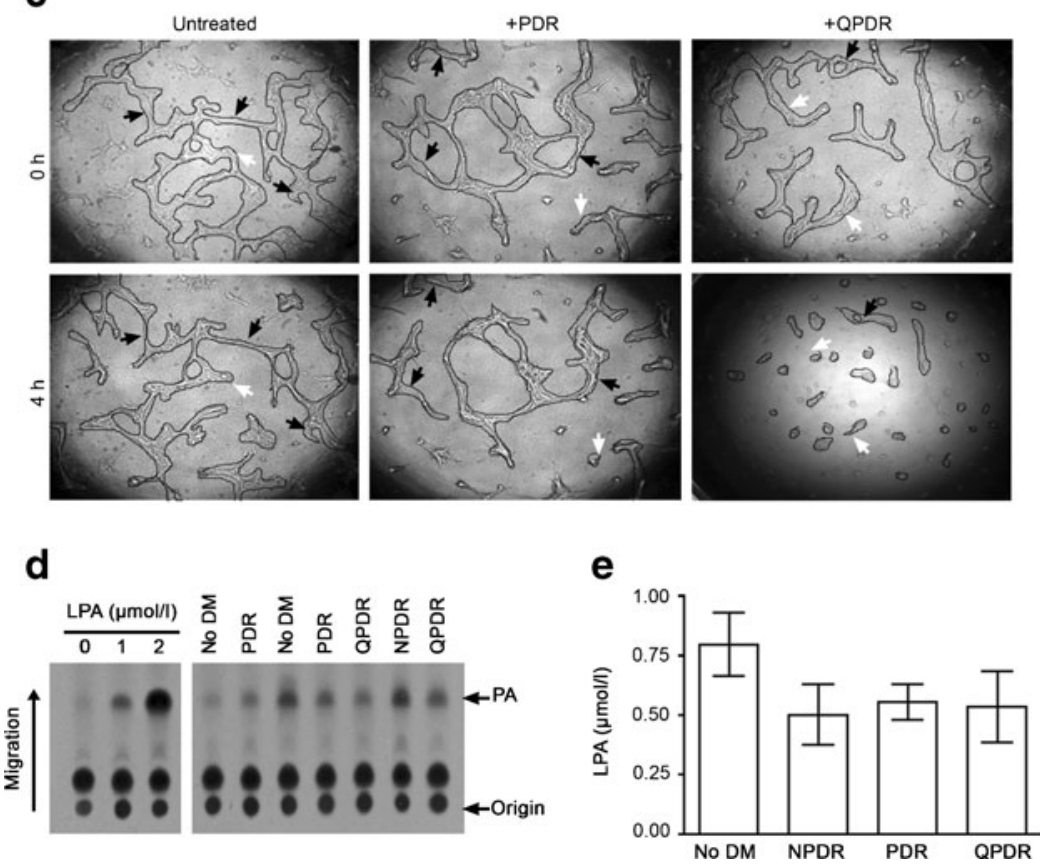
there was as much LPA in PDR vitreous as in vitreous that efficiently promoted regression (Fig. 4d,e). The concentrations of LPA for the different stages of DR were: NPDR $0.50 \pm 0.15 \mu \mathrm{mol} / \mathrm{l}$; PDR $0.55 \pm 0.07 \mu \mathrm{mol} / \mathrm{l}$; and QPDR $0.53 \pm 0.18 \mu \mathrm{mol} / \mathrm{l}$. These data indicate that vitreous from patients with PDR failed to promote regression, despite the fact that it contained sufficient LPA.

PDR vitreous antagonised LPA-mediated regression We sought to further investigate how PDR vitreous influenced LPA-mediated regression. To this end, we determined if PDR vitreous influenced regression when driven by exogenously added LPA. As expected, tubes were stable until LPA was added, which then induced their regression (Fig. 5). When PDR vitreous was added together with LPA, regression was inhibited (Fig. 5). We conclude that PDR vitreous not only prevents regression driven by LPA present in PDR (Fig. 4), but also by exogenously added LPA (Fig. 5).

DR alters the composition of vitreous; for instance, VEGF-A is higher in PDR vitreous than in vitreous from patients with other stages of DR [7,34]. These previous observations raised the possibility that VEGF-A in PDR vitreous prevented LPA-mediated regression. However, this did not turn out to be the case, as increasing the concentration of VEGF-A to $25 \mathrm{ng} / \mathrm{ml}$, which is well in excess of the average level present in PDR vitreous $(8.8 \mathrm{ng} / \mathrm{ml})$ [7] did not prevent LPA-mediated regression (Fig. 5). This result is consistent with our previous observations that LPA-mediated regression proceeds even in the presence of serum and proangiogenic factors such as VEGF-A and/or basic fibroblast growth factor (bFGF) $[25,29]$. Unlike regression that inevitably results when cells die on neutralisation of growth factors, LPA-induced regression is a deliberate response induced by signalling events such as rho-associated protein kinase (ROCK) activation [28, 35].

An additional version of the tube assay was used to further test the ability of PDR vitreous to prevent regression. When cultured in $10 \%$ (vol./vol.) serum, tube regression is driven by the combination of endogenously produced LPA and LPA from serum $[25,35]$. While NPDR and QPDR vitreous had no effect on spontaneous regression that occurs under these conditions (perhaps because there was sufficient LPA to drive this response), PDR vitreous blocked regression (Fig. 6). This additional experimental setting confirmed that PDR vitreous prevents LPA-mediated regression.

LPA resistance, which was induced by PDR vitreous, could be overcome pharmacologically To begin to understand the mechanism by which PDR vitreous antagonised LPAmediated regression, we considered if it was dependent on the ROS, Src family kinase (SFK), ERK (RSE) pathway, which antagonises signalling events (i.e. ROCK activity) required for LPA-dependent outcomes [26, 28, 36]. For a

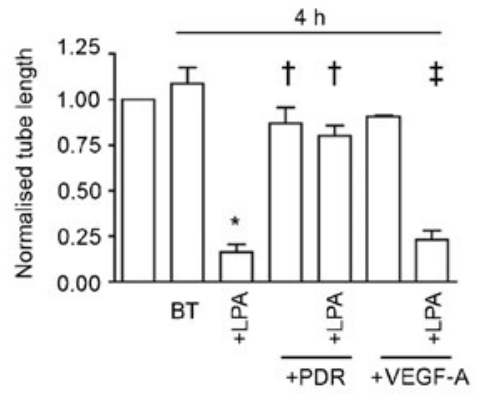

b
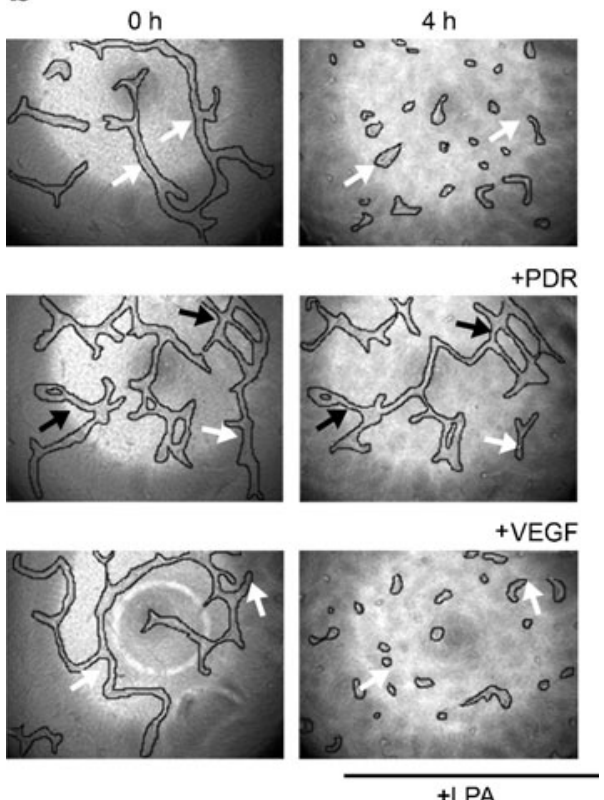

Fig. 5 PDR vitreous inhibited exogenous LPA-induced tube regression. The collagen sandwich tube assay was performed with parental BRECs in low serum, conditions under which exogenous LPA is necessary to promote regression [25]. (a) Addition of LPA $(1 \mu \mathrm{mol} / \mathrm{l})$ induced regression, and this response was inhibited when the LPA was added together with PDR vitreous $(n=3)$. In contrast, LPA-induced regression was not diminished by VEGF-A $(25 \mathrm{ng} / \mathrm{ml})$. Data shown are means \pm SEM; ${ }^{*} p<0.05$ compared with buffer treatment $(\mathrm{BT}) ;{ }^{\dagger} p<0.05$ compared with exogenous LPA treatment; ${ }^{\ddagger} p<0.05$ VEGF-A treatment vs VEGF-A + LPA group. Normalised tube length is the ratio of final and initial tube lengths. (b) Photos of representative tubes

instance, high-glucose treatment of primary retinal endothelial cells induces a state of LPA unresponsiveness, which can be overcome by inhibiting members of the RSE pathway [26].

We considered if this mechanism was the basis for PDRvitreous-induced non-responsiveness to LPA. First, we tested the ability of ROS to induce resistance to exogenous LPA in tubes organised from endothelial cells (Fig. 7a). Indeed, the presence of hydrogen peroxide inhibited the LPA-driven regression of BREC tubes (Fig. 7a). This result established that at least one component of the RSE (ROS, SFK, ERK) pathway was driving LPA resistance. Therefore, in our next 


\section{a}

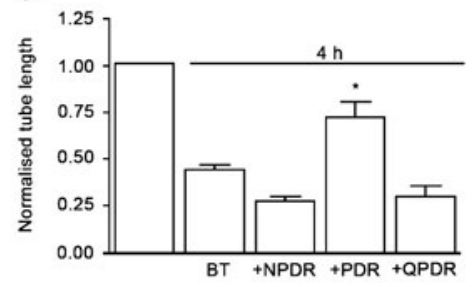

b
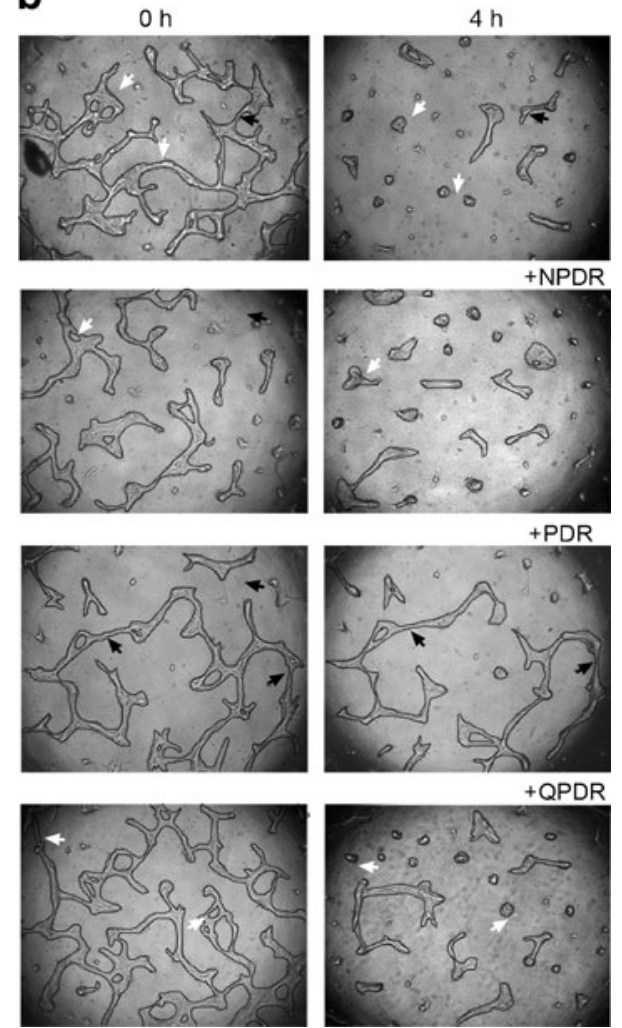

+ PDR
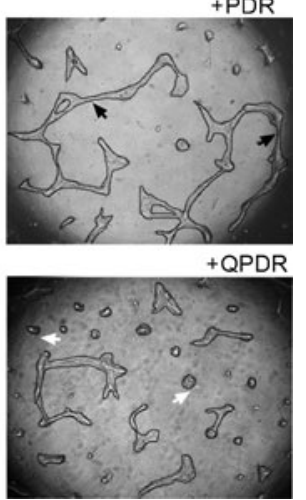

Fig. 6 Vitreous from PDR patients prevented spontaneous regression. The collagen sandwich tube assay was performed in $10 \%$ (vol./vol.) serum, conditions under which tubes regress spontaneously because of the combined effect of LPA produced by cells and present in serum [25]. The indicated type of vitreous was added at $12 \mathrm{~h}$, and its effect determined $4 \mathrm{~h}$ later. Whereas the effect of NPDR and QPDR vitreous was not statistically significant, PDR vitreous prevented regression ${ }^{*} p<0.05$ compared with no treatment). Normalised tube length is the ratio of final and initial tube lengths. Three samples of each DR stage were analysed

series of experiments we tested the effect of chemical inhibitors targeting each component of this pathway. As shown in Fig. 7b, c, NAC, a well-known antioxidant, restored responsiveness to LPA in PDR vitreous samples. Importantly, this result was also observed with other chemically distinct antioxidants (ESM Fig. 2). In support of an RSE role, we observed that inhibitors of SFKs or the ERK pathway overcame PDR-vitreous-induced resistance to LPA-dependent regression (Fig. 7b,c). These observations indicate that the PDR-vitreous-mediated non-responsiveness to LPA was dependent on the RSE a

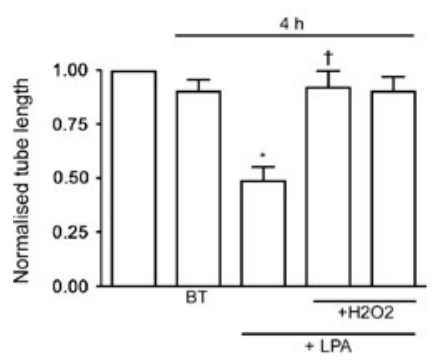

C

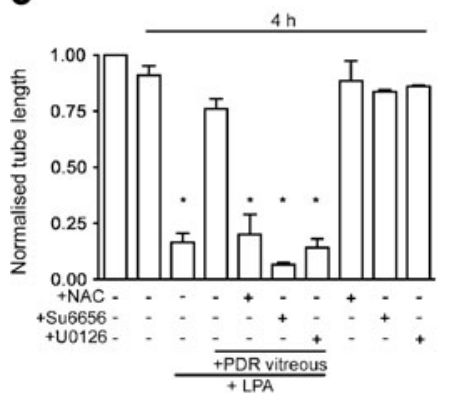

b
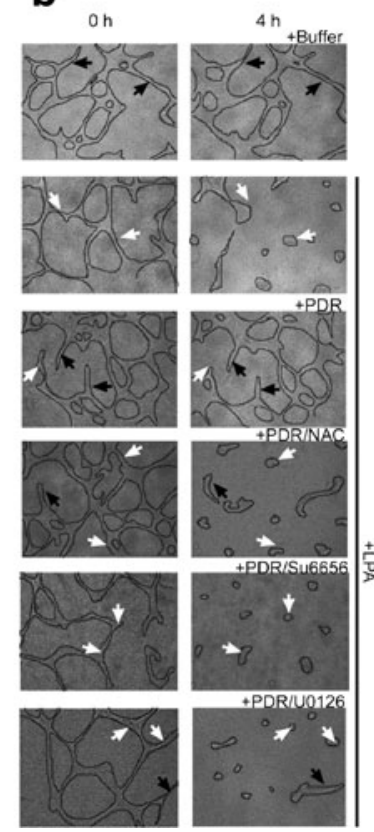

Fig. 7 ROS is a key mediator of LPA resistance that was induced by PDR vitreous, and which could be overcome pharmacologically by targeting the RSE pathway. (a) Exogenous LPA was added to BREC tubes in the presence or absence of hydrogen peroxide. As expected, LPA induced significant tube regression $\left(^{*} p<0.05\right)$. In contrast, hydrogen peroxide significantly inhibited regression in response to LPA $\left({ }^{\dagger} p<0.05\right.$ vs LPA-treated tubes). (b,c) Same experimental conditions as in Fig. 6, except with the indicated additions. LPA $(1 \mu \mathrm{mol} / \mathrm{l})$, NAC

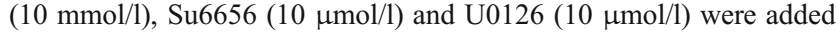
either alone or in combination with the same PDR vitreous. Inhibitors of the RSE pathway enabled LPA to promote regression in the presence of PDR vitreous. The data are means $\pm \operatorname{SEM}(n=4)$. Normalised tube length is the ratio of final and initial tube lengths. ${ }^{*} p<0.05$ vs control group

pathway, and suggested that the underlying mechanism involved a decreased ability of retinal endothelium to respond to LPA.

\section{Discussion}

The three major findings in this report are: (1) vitreous had intrinsic regression activity that was largely dependent on LPA; (2) as patients developed PDR, vitreous lost its ability to promote regression, even though the level of LPA was unchanged; and (3) the unresponsiveness to LPA could be overcome with pharmacological inhibitors of the RSE pathway.

In light of the relevance of this work to PDR, an important issue is the translatability of our findings. A clear example of this is the critical role that ROS play in inhibiting the endothelial response to LPA. Clinical studies suggest that elevated ROS levels are detected in PDR vitreous compared with control samples [24], and increased oxidative stress has been reported in rodent models of DR [37]. In addition, ROS 
production has been shown to promote retinal cell survival [38]. Despite these findings, the results of clinical trials of antioxidant therapy are contradictory. Studies with limited numbers of patients suggest that antioxidant therapy with dobesilate and pycnogenol delay DR progression [39-41]. In contrast, others have found no association between antioxidant therapy and decreased progression of DR [42, 43]. The reason for these conflicting results is elusive. Important factors to consider are the damage already sustained by the retina prior to beginning antioxidant therapy, and the dose of antioxidant that reaches the eye. Nevertheless, basic science studies consistently point to a critical role of ROS, and warrant further investigation.

One of the questions that emerge from our findings relates to how the largely acellular PDR vitreous induces a state of non-responsiveness to LPA. In light of the fact that it can be overcome with inhibitors of the RSE pathway, our working hypothesis is that PDR vitreous contains agents secreted by retinal cells that are capable of activating this pathway. We are currently testing this hypothesis and attempting to identify such agents.

Multiple studies indicate that in certain settings ATX and LPA promote angiogenesis [21, 22, 44, 45]. Our results indicate that there are conditions in which ATX and LPA induce regression of unstable vascular beds, such as the hyaloid [25]. We propose that in unstable vessels, LPAinitiated signalling stimulates cell migration, resulting in the disorganisation of vessels [27].

Early reports documenting the regression of blood vessels indicated that apoptosis was the underlying cause of regression. Acute cessation of a pro-angiogenic stimulus was associated with apoptosis of the endothelial cells constituting immature blood vessels [46, 47]. LPA-mediated regression proceeds by a distinct mechanism [25, 35]. Thus, there are multiple modes to instruct regression of blood vessels.

One implication of our findings is that PDR pathogenesis involves not only an increase in the vitreal level of proangiogenic agents, but also a decline in the responsiveness to vitreal regression factors such as LPA. Enforcing LPA responsiveness may be an approach to protect patients from developing PDR and a novel strategy to enhance the effectiveness of anti-VEGF-A-based therapies.

\footnotetext{
Acknowledgements $\mathrm{K}$. Kume from the Department of Medicine, $\mathrm{Ku}-$ mamoto University, Japan, kindly donated the plasmid encoding LPAAT. The VEGF-A used for this study was provided by the BRB Preclinical Repository of the National Cancer Institute, Frederick, MD, USA.

Funding Grant Numbers and Sources of Support: I-2008-905 and EY018344 from JDRF and National Institutes of Health, respectively, to A. Kazlauskas; Pew Latin American Fellows Program in the Biomedical Sciences, American Diabetes Association Mentor-based Minority Fellowship 7-09-MI-04 and Fundacion Mexico en Harvard to J. Aranda.
}

Duality of interest The authors confirm that there is no duality of interest associated with this manuscript.

Contribution statement JA and AK designed experiments and wrote the manuscript. JA performed all experiments except for quantification of lysophosphatidic acid in clinical specimens, which was done by RM, who also provided critical input. LPA and PS isolated all clinical specimens, and contributed to the analysis and interpretation of data. All authors contributed to the drafting or revising of the manuscript and approved its final version.

\section{References}

1. International Diabetes Federation (2011) IDF Diabetes Atlas, 5th edn. IDF, Brussels. Available from http://www.idf.org/ diabetesatlas, accessed 20 Feb 2013

2. Antonetti DA, Klein R, Gardner TW (2012) Diabetic retinopathy. N Engl J Med 366:1227-1239

3. Cheung N, Mitchell P, Wong TY (2010) Diabetic retinopathy. Lancet 376:124-136

4. Engerman RL, Kern TS (1995) Retinopathy in animal models of diabetes. Diabetes Metabol Rev 11:109-120

5. Aiello LP, Gardner TW, King GL et al (1998) Diabetic retinopathy. Diabetes Care 21:143-156

6. Gariano RF, Gardner TW (2005) Retinal angiogenesis in development and disease. Nature 438:960-966

7. Aiello LP, Avery RL, Arrigg PG et al (1994) Vascular endothelial growth factor in ocular fluid of patients with diabetic retinopathy and other retinal disorders. N Engl J Med 331:1480-1487

8. Watanabe D, Suzuma K, Matsui S et al (2005) Erythropoietin as a retinal angiogenic factor in proliferative diabetic retinopathy. N Engl J Med 353:782-792

9. Frank RN (2004) Diabetic retinopathy. N Engl J Med 350:48-58

10. Silva PS, Cavallerano JD, Sun JK, Aiello LM, Aiello LP (2010) Effect of systemic medications on onset and progression of diabetic retinopathy. Nat Rev Endocrinol 6:494-508

11. Cury CE Jr, Rodrigues EB, Meyer CH, Farah ME (2009) VEGF inhibitors and vitrectomy for diabetic vitreoretinopathy. Dev Ophthalmol 44:69-81

12. Iacono P, Battaglia Parodi M, Bandello F (2010) Antivascular endothelial growth factor in diabetic retinopathy. Dev Ophthalmol 46:39-53

13. Adamis AP, Altaweel M, Bressler NM et al (2006) Changes in retinal neovascularization after pegaptanib (Macugen) therapy in diabetic individuals. Ophthalmology 113:23-28

14. Mirshahi A, Roohipoor R, Lashay A, Mohammadi SF, Abdoallahi A, Faghihi H (2008) Bevacizumab-augmented retinal laser photocoagulation in proliferative diabetic retinopathy: a randomized double-masked clinical trial. Eur J Ophthalmol 18:263-269

15. Preis I, Langer R, Brem H, Folkman J (1977) Inhibition of neovascularization by an extract derived from vitreous. Am J Ophthalmol 84:323-328

16. Felton SM, Brown GC, Felberg NT, Federman JL (1979) Vitreous inhibition of tumor neovascularization. Arch Ophthalmol 97:1710-1713

17. Lutty GA, Thompson DC, Gallup JY, Mello RJ, Patz A, Fenselau A (1983) Vitreous: an inhibitor of retinal extract-induced neovascularization. Invest Ophthalmol Vis Sci 24:52-56

18. Aoki J (2004) Mechanisms of lysophosphatidic acid production. Semin Cell Dev Biol 15:477-489

19. Moolenaar WH, van Meeteren LA, Giepmans BN (2004) The ins and outs of lysophosphatidic acid signaling. Bioessays 26:870-881

20. Nakanaga K, Hama K, Aoki J (2010) Autotaxin - an LPA producing enzyme with diverse functions. J Biochem 148:13-24 
21. van Meeteren LA, Ruurs P, Stortelers C et al (2006) Autotaxin, a secreted lysophospholipase D, is essential for blood vessel formation during development. Mol Cell Biol 26:5015-5022

22. Nam SW, Clair T, Kim YS et al (2001) Autotaxin (NPP-2), a metastasis-enhancing motogen, is an angiogenic factor. Cancer Res 61:6938-6944

23. Nam SW, Clair T, Campo CK, Lee HY, Liotta LA, Stracke ML (2000) Autotaxin (ATX), a potent tumor motogen, augments invasive and metastatic potential of ras-transformed cells. Oncogene 19:241-247

24. Yeh PT, Yang CM, Huang JS et al (2008) Vitreous levels of reactive oxygen species in proliferative diabetic retinopathy. Ophthalmology 115(734-737):e731

25. Im E, Motiejunaite R, Aranda J et al (2010) Phospholipase Cgamma activation drives increased production of autotaxin in endothelial cells and lysophosphatidic acid-dependent regression. Mol Cell Biol 30:2401-2410

26. Aranda J, Motiejunaite R, Im E, Kazlauskas A (2012) Diabetes disrupts the response of retinal endothelial cells to the angiomodulator lysophosphatidic Acid. Diabetes 61:1225-1233

27. Im E, Venkatakrishnan A, Kazlauskas A (2005) Cathepsin B regulates the intrinsic angiogenic threshold of endothelial cells. Mol Biol Cell 16:3488-3500

28. Im E, Kazlauskas A (2007) Src family kinases promote vessel stability by antagonizing the Rho/ROCK pathway. J Biol Chem 282:29122-29129

29. Im E, Kazlauskas A (2006) Regulating angiogenesis at the level of PtdIns-4,5-P(2). EMBO J 25:2075-2082

30. Saulnier-Blache JS, Girard A, Simon MF, Lafontan M, Valet $P$ (2000) A simple and highly sensitive radioenzymatic assay for lysophosphatidic acid quantification. J Lipid Res 41:1947-1951

31. Eichholtz T, Jalink K, Fahrenfort I, Moolenaar WH (1993) The bioactive phospholipid lysophosphatidic acid is released from activated platelets. Biochem J 291(Pt 3):677-680

32. Nagasaki T, Gundersen GG (1996) Depletion of lysophosphatidic acid triggers a loss of oriented detyrosinated microtubules in motile fibroblasts. J Cell Sci 109(Pt 10):2461-2469

33. Park EY, Kazlauskas A (2012) Primary human endothelial cells secrete agents that reduce responsiveness to lysophosphatidic acid (LPA). Biosci Rep 32:393-400

34. Ogata N, Nishikawa M, Nishimura T, Mitsuma Y, Matsumura M (2002) Unbalanced vitreous levels of pigment epithelium-derived factor and vascular endothelial growth factor in diabetic retinopathy. Am J Ophthalmol 134:348-353

35. Im E, Kazlauskas A (2006) New insights regarding vessel regression. Cell Cycle 5:2057-2059

36. Mavria G, Vercoulen Y, Yeo M et al (2006) ERK-MAPK signaling opposes Rho-kinase to promote endothelial cell survival and sprouting during angiogenesis. Canc Cell 9:33-44

37. Kowluru RA, Odenbach S (2004) Role of interleukin-1beta in the development of retinopathy in rats: effect of antioxidants. Invest Ophthalmol Vis Sci 45:4161-4166

38. Groeger G, Mackey AM, Pettigrew CA, Bhatt L, Cotter TG (2009) Stress-induced activation of Nox contributes to cell survival signalling via production of hydrogen peroxide. J Neurochem 109:1544-1554

39. Garay RP, Hannaert P, Chiavaroli C (2005) Calcium dobesilate in the treatment of diabetic retinopathy. Treat Endocrinol 4:221-232

40. Kowluru RA (2003) Effect of reinstitution of good glycemic control on retinal oxidative stress and nitrative stress in diabetic rats. Diabetes 52:818-823

41. Spadea L, Balestrazzi E (2001) Treatment of vascular retinopathies with Pycnogenol. Phytother Res PTR 15:219-223

42. Mayer-Davis EJ, Bell RA, Reboussin BA, Rushing J, Marshall JA, Hamman RF (1998) Antioxidant nutrient intake and diabetic retinopathy: the San Luis Valley Diabetes Study. Ophthalmology 105:2264-2270

43. Millen AE, Klein R, Folsom AR, Stevens J, Palta M, Mares JA (2004) Relation between intake of vitamins $\mathrm{C}$ and $\mathrm{E}$ and risk of diabetic retinopathy in the Atherosclerosis Risk in Communities Study. Am J Clin Nutr 79:865-873

44. Ptaszynska MM, Pendrak ML, Bandle RW, Stracke ML, Roberts DD (2008) Positive feedback between vascular endothelial growth factor-A and autotaxin in ovarian cancer cells. Mol Canc Res $6: 352-363$

45. Rivera-Lopez CM, Tucker AL, Lynch KR (2008) Lysophosphatidic acid (LPA) and angiogenesis. Angiogenesis 11:301-310

46. Ausprunk DH, Falterman K, Folkman J (1978) The sequence of events in the regression of corneal capillaries. Lab Invest 38:284 294

47. Kenyon BM, Voest EE, Chen CC, Flynn E, Folkman J, D'Amato RJ (1996) A model of angiogenesis in the mouse cornea. Invest Ophthalmol Vis Sci 37:1625-1632 